\title{
USE OF 3D SIMULATION TO DESIGN THEORETICAL AND REAL PIPE INSPECTION MOBILE ROBOT MODEL
}

\author{
Leszek BARANOWSKI*, Michał SIWEK ${ }^{*}$ \\ ${ }^{*}$ Faculty of Mechatronics and Aviation, Military University of Technology, ul. Urbanowicza 2, 00-908 Warszawa, Poland \\ leszek.baranowski@wat.edu.pl, michal.siwek@wat.edu.pl
}

received 10 April 2017, revised 24 September 2018, accepted 27 September 2018

\begin{abstract}
The main aim of the paper is to present the process of design pipe inspection mobile robot by using 3D simulations. Next methods and processes of making designed components was described. Finally, functional tests of a constructed real robot model such as speed tests, inclined pipe test was carried out. The robot was specifically designed to inspect sewer pipelines. The mobile robot is equipped with a vision system. The structure of the pipe inspection robot allows adjustments to the geometrical parameters of the robot to suit the sewer pipes diameters by using in the construction of a pneumatic system with an actuator.
\end{abstract}

Key words: Pipe Inspection Robot Model, Inspection Robot, Mobile Robot 3D Simulation

\section{INTRODUCTION}

The use of inspection robots for working in places difficult to access is a very popular developing trend in mobile robotics. A large percentage of all designed and built mobile robots are being used for specific inspection tasks. One of the hardest places to access are the pipelines, specifically sewer pipelines, where there is a high probability of blockage. The aspect of keeping clean and efficient is very important for proper functioning of the pipelines. In view of difficult access to the pipelines, small diameters and large pipe lengths mobile pipe inspection robots are commonly used tool to keep the correct flow and tightness control. The use of mobile robots to conduct an analysis of the pipeline state of repair and defects identification allows very fast localization and removal of damage without the need to dig out the pipe. The use of modern simulation tools allows the engineers to design and build more advanced and more functional structures of mobile robots. Development of complex structures of mechatronic devices takes a special mechatronic design process. This process consists in designing at the same time a CAD model, computer simulations of device operation, electrical connections design and software. Use of the mechatronic devices process for development of mobile robot is described in Baranowski et al. (2017), Ciszewski et al. (2014), Fu et al. (2008), Yuan et al. (2010) and Erosoy and Yilmaz, (2008). The authors present an innovative solution using a pneumatic actuator as an element to change the robot's configuration and to adapt the construction to the selected pipe diameter. The use of a pneumatic system allows elasticity of the robot structure in the joint, which is an advantage in the case of overcoming obstacles in the rump such as dents, cracks and faults, which cannot be obtained when using a structure with servos as well as in the case described in Giergiel et al. (2015) and Szewczyk (2017). However, supplying the pneumatic system with an internal compressed air tank limits the possibility to con- figure the robot which is a disadvantage of this idea. Next requirement for the pneumatic system was the necessity of maintaining small dimensions of the robot's structure in relation to a quite large range of pipe (for example, the design described in Szewczyk (2017) allows changing the pipe diameter by only $40 \mathrm{~mm}$ in relation to the minimum diameter). The robot described in this paper was designed for work in sewer pipes of single-family households, where the range of diameters from $160 \mathrm{~mm}$ to $300 \mathrm{~mm}$ is most often used. In addition, the sewerage environment is a difficult environment for the robot due to the large amount of moisture and pollution. The use of less complicated systems and fewer electronic systems in the robot's design results in a long failure-free operation of the device. Fig. 1 shows the CAD model of the pipe inspection robot, which was conceptually described in Panasiuk and Siwek (2016).

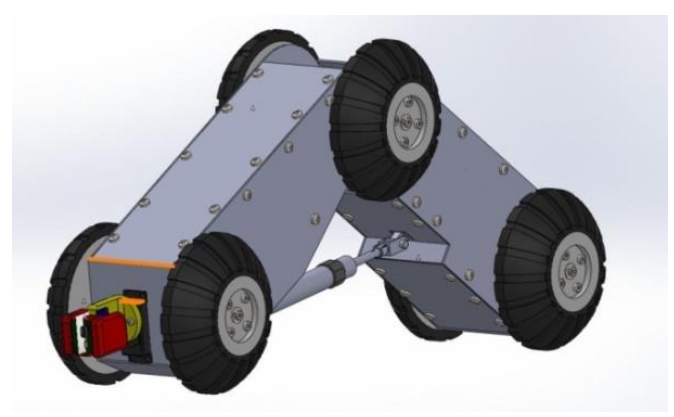

Fig. 1. The pipe inspection robot $C A D$ model

The first step to implement the project is preparing project requirements. Driving and functional requirements were developed based on the analysis of the pipe inspection robot design, commercial mobile robot structure, the constructor own observation and chosen robot working environmental requirement for the robot characteristics. 
The design of pipe inspection robot should allow:

- inspection of sewer pipelines of diameters from $160 \mathrm{~mm}$ to $300 \mathrm{~mm}$;

- inspection of ground and underground sewer pipelines;

- inspection of min $50 \mathrm{~m}$ sewer pipe length;

- estimation of real time pipelines state of repair;

- inspection state of repair independently of temperature and weather conditions;

- inspection of inclined pipelines.

Pipe inspection robot technical requirements are as follows:

- the inspection system should consist of mobile platform and operator station;

- the mobile platform and the operator station should be equipped with their own supplies;

- the robot structure should allow an easy access to the exploitation connections of the robot;

- the robot structure should allow manual removal from the pipelines;

- the system should signal a low battery level;

- the robot structure should provide easy service.

Pipe inspection robot reliability requirements are as follows

- ensure trouble-free device operation;

- ensure video data quality required for inspection;

- use of the inspection system by a trained operator;

- service at designated intervals.

\section{THE PIPE INSPECTION ROBOT CAD MODEL}

The pipe inspection robot structure is shown in the form of Product Breakdown Structure. PBS is part of the PRINCE2 project management. PBS is a tool used to plan, present results and project evaluation. It is based on a detailed division of the project. In this method it is important not to miss any component parts.

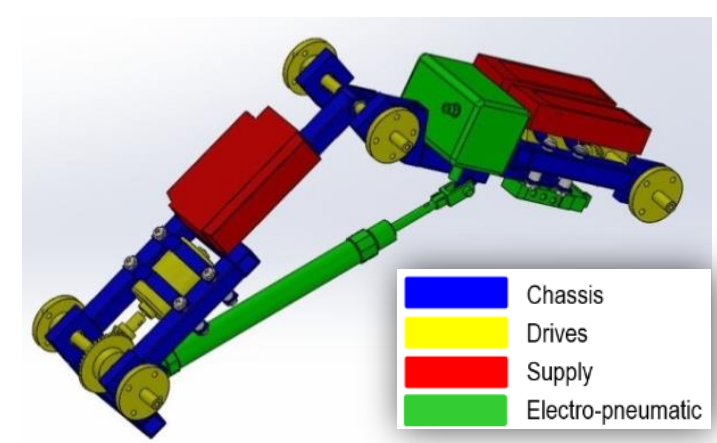

Fig. 2. The inspection robot colour coding

The PBS diagram shows 6 main branches denoting the various pipe inspection system sections: chassis, drives, supply, electro-pneumatic, control system and vision system. Fig. 2 shows the robot section colour coding, consistent with PBS diagram.

\subsection{The chassis design}

The structure of the chassis is two-part. Front part and back part are connected in the centre of chassis by one degree of freedom joint. The concept of using an articulated frame was created after analysis of the snake robots described in Liljeback et al. (2012), Trebuna et al. (2016) and Brunete et al. (2012). The building material is $15 \times 15 \mathrm{~mm}$ square steel profile. The chassis includes ball bearing mounting, DC motors mounting and other components mounting. Use of pneumatic actuator allows bending the chassis in the joint. The possibility of lifting the central pair of wheels allows a change in the robot configuration and adaptation to pipe diameter. Fig. 3a shows basic chassis configuration, Fig. 3b shows bent chassis configuration.

a)

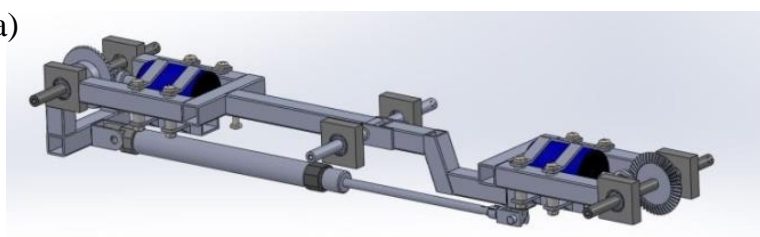

b)

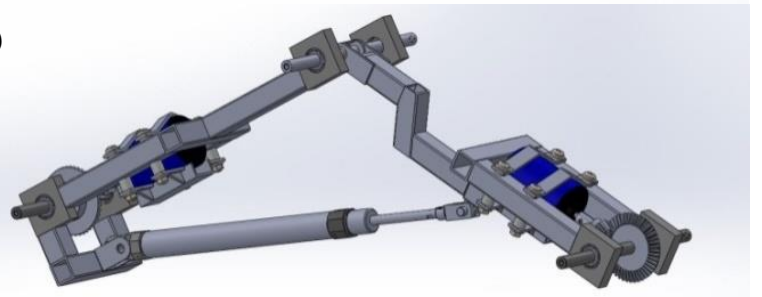

Fig. 3. The chassis configuration: a) basic, b) bent

Based on the chassis CAD model analysis in SOLIDWORKS 2013 the possibility of adapting configuration of the robot to suit the sewer pipes diameter has been examined. Final dimension of the wheels has been chosen and put into final modification of the chassis. The final effect of this simulation confirms feasibility of adapting robot configuration to pipe of diameters from $160 \mathrm{~mm}$ to $300 \mathrm{~mm}$.

\subsection{The drive system design}

The analysis of the pipe inspection mobile robots design indicates a huge versatility of the drive system. For this project 6wheeled drive system has been chosen. Wheels are combined in pairs, front pair and rear pair monitored by $\mathrm{DC}$ motors. The centre pair of wheels is rotating freely. It is an additional point of support during the inspection of pipelines with diameter above $160 \mathrm{~mm}$. The torque is transmitted to the wheels by the clutch and bevel gear with a 3:1 ratio. Driving axle is mounted in ball bearings. The wheels are fastened to axles by special fastener. Fig. 4 shows the design of the drive system.

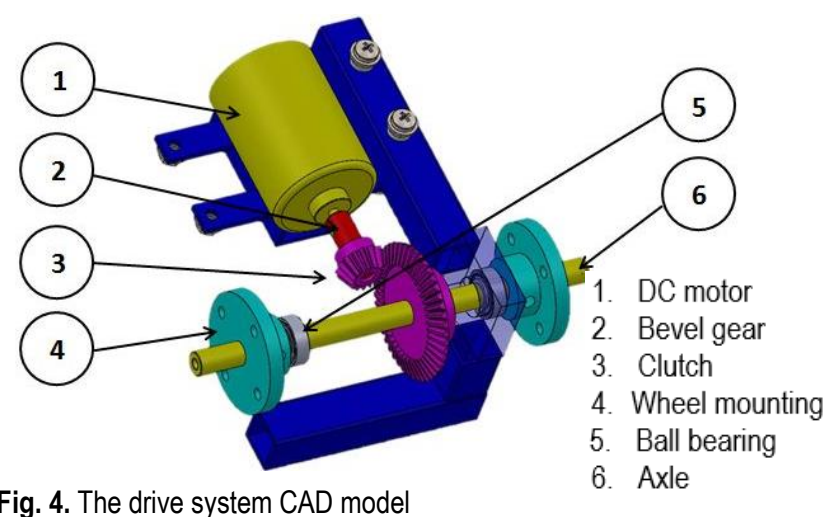

Fig. 4. The drive system CAD model 


\subsection{The wheels design}

Considering required diameter range used in SOLIDWORKS 2013 a special wheel model was developed, it allows stable movement in the pipelines shown in Fig. 5. Wheels were designed as two-part and printed on the 3D printer. Hard body-rim was printed from ABS (Acrylonitrile Butadiene Styrene) which is an extremely durable, impact resistant filament, ideal for 3D printing of functional prototypes. ABS is very light and very dimensionally stable. The rim has a space for special connector for fixing the wheel on the axle. The second part was the rubber tire printed from SOFT PLA, which is very elastic and resembles rubber with its properties. This material practically does not shrink during 3D printing. Both elements have been glued permanently.
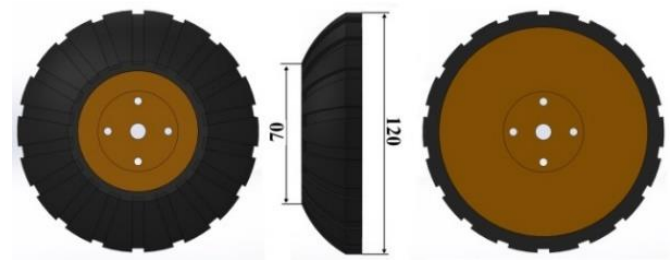

Fig. 5. Wheels CAD model

Wheel section has a variable diameter. The diameter decreases towards the outside of the robot. This solution ensures larger area of contact surface of the tire with the pipe. Large contact surface provides stable positioning of the robot in the pipe. The tires are equipped with tread to drain liquid from contact surface. Such a solution allows to increase the friction of the tire by the pipe, which is especially necessary during inspections of inclined pipelines.

\subsection{The air pressure tank design}

To increase the mobility of the inspection system, pneumatic system should have its own air pressure source. Air pressure tank dedicated and specially designed for this project is placed in the rear part of the chassis. The tank is designed as a rectangular block with pneumatic connectors. The tank is easy to fix to the robot's chassis. Building material is a square steel pipe of section $50 \times 50 \mathrm{~mm}$ and $4 \mathrm{~mm}$ thickness of the wall. The tank provides enough compressed air and air pressure for one full cycle of the actuator. One full cycle of actuator pistons the rod out, keeps the position for required time, then returns the piston rod to the starting position.

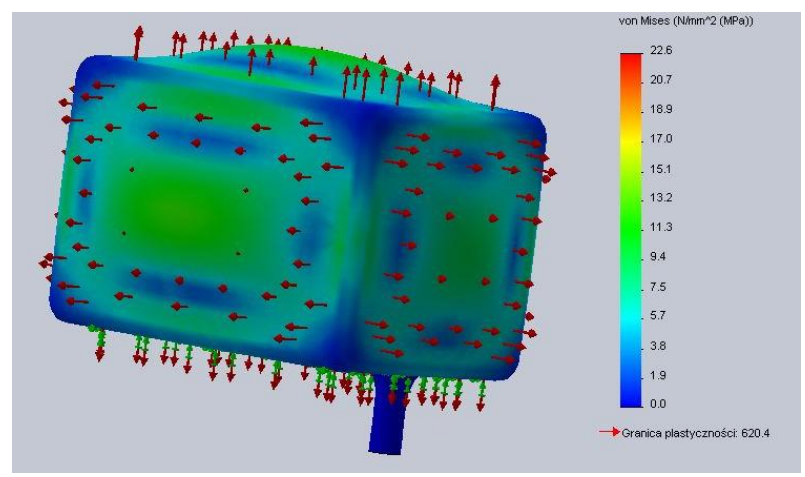

Fig. 6. Mechanical stresses simulation
Replenishing the air is provided by car valve and pump or compressor. SOLIDWORKS 2013 was used to conduct the tank technical analysis shown in Fig. 6 (mechanical stresses simulation) and Fig. 7 (simulation of deformation). The tank requires a load of 7 bar pressure for proper operation of the electropneumatic system. The analysis shows that $4 \mathrm{~mm}$ thickness of wall withstands the required pressure conditions.

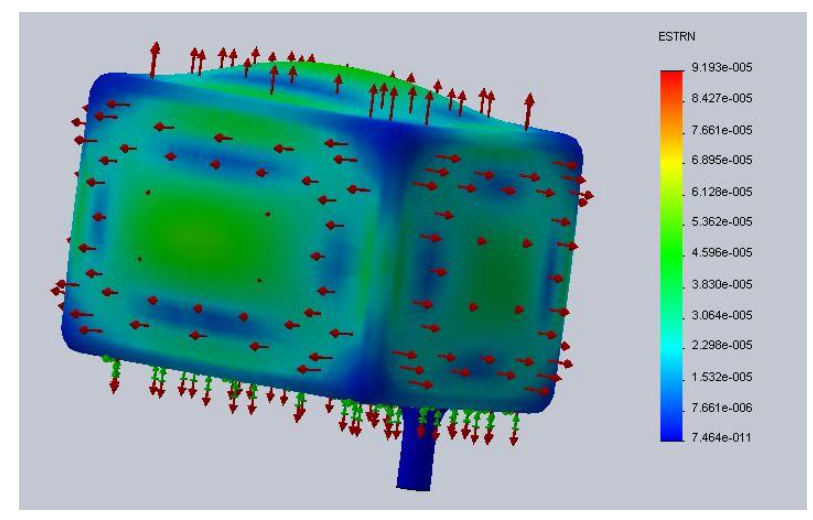

Fig. 7. Simulation of deformation

\section{BUILDING THE PIPE INSPECTION ROBOT}

The first step to build the pipe inspection robot real model is preparing chassis component and its structure. Next step is to integrate the chassis and the actuator, to attach the drive systems to the air pressure tank. After that, the robot housing and wheel are made.

The robot chassis is the main part of mobile robot, it keeps all the other components. The chassis is adjusted for the robot functionality. Based on the drawing in SOLIDWORKS 2013 the chassis component CAD model was developed. The chassis component parts were combined by a shield metal arc welding. The final step was to polish the moulded parts and to paint the chassis. The assembled chassis parts are shown in Fig. 8.

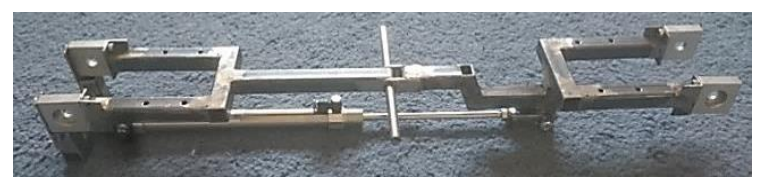

Fig. 8. The robot chassis basic configuration

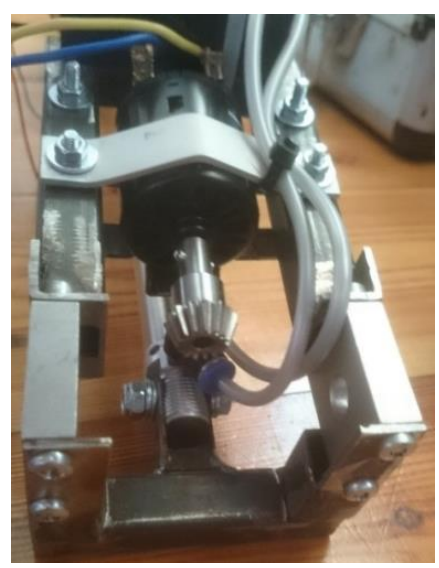

Fig. 9. The robot drive system 
The last step in the robot chassis construction was the attachment of the drive system. The ball bearings were placed in the special bearing housing fixed to the robot chassis. Then the axles were mounted with the use of circlips. DC motor made of channel of section $15 \times 20 \mathrm{~mm}$ was also mounted. Position of the DC motor is regulated by screws in a nylon bush. This solution allows adjustment of the DC motor position relative to axis position. The DC motor position is stabled by aluminium clamp. The robot drive system is shown in Fig. 9.

Based on the CAD model of the robot, a tank for pressured air has been made. The building material is a square pipe of section $50 \times 50 \mathrm{~mm}$. The air pressure tank parts were combined by shield metal arc welding. Next two holes for attachment have been drilled. After the pressure tank was made, a leak test was carried out. The tank was filled with air at pressure 4.5 bars and immersed in water. Then, for 30 minutes (robot operation time on fully charged batteries), the air pressure in the tank was observed as well as the appearance of bubbles signalling the leak. After 30 minutes a drop-in pressure of 0.1 bar (accuracy of the measurement instrument) was observed, which was an acceptable value. The test was repeated several times regarding higher pressure values not greater than 7 bars. The leak tests showed that the air pressure tank maintains the required pressure of 4.5 bars. The leak test is shown in Fig. 10.

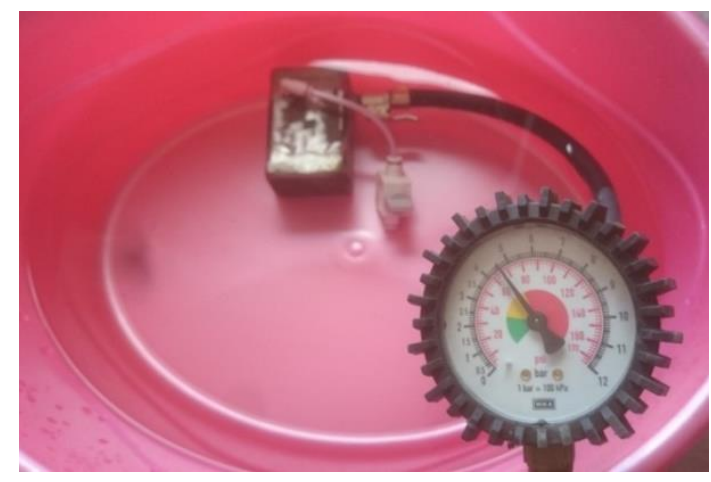

Fig. 10. The air pressure tank leak test

The mobile robot housing was produced based on the robot CAD model using laser cutting and laser welding. The robot housing parts were cut from the metal sheet with a thickness of $1 \mathrm{~mm}$. Then, after initial connection the robot housing was welded with laser by FANUC industrial robot. Fig. 11 shows the welded robot housing part.

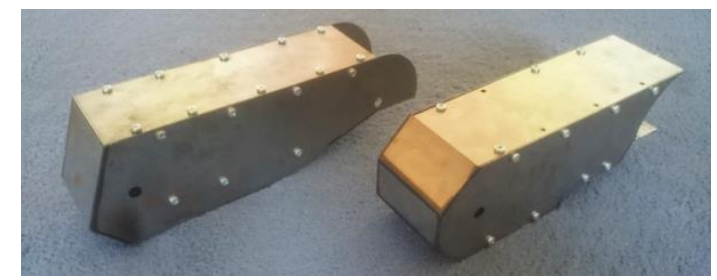

Fig. 11. The robot housing

The set of wheels was printed on a 3D printer. First, test prints of the tires and rims were made to select the optimal printing parameters. Next, a whole set of wheels was printed. Set of wheels is shown in Fig. 12.

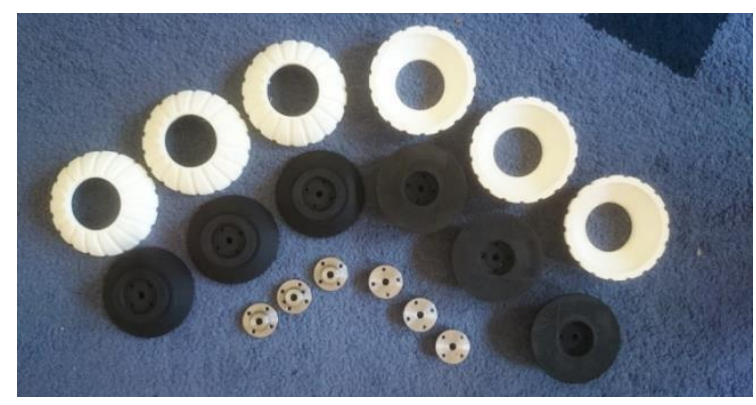

Fig. 12. The set of wheels

The vision system was built based on the studies by Kawasue and Komatsu, (2013), Martinez-Gomez et al. (2014), Ono and Kato (2010) and Hansen et al. (2015). The rotary head with a HD camera was printed by Zortrax 3D printer. Building material is ZABS. Next, a camera and servos are added to the printed head parts. The rotary head parts do not include additional transfer torque system from servos.

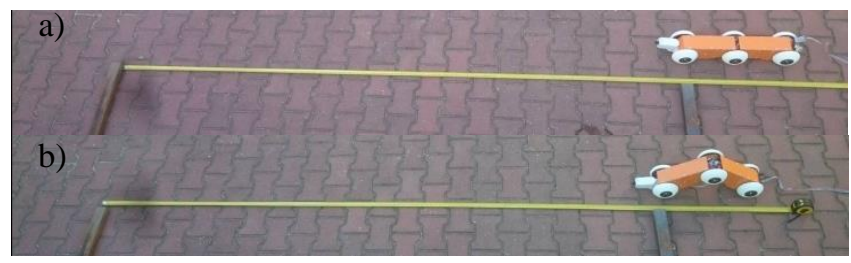

Fig. 13. The speed test: a) basic robot configuration, b) bent robot configuration

The final step of the building was to assemble components part and integrate the robot control system. The real model of pipe inspection has been tested in a working environment. The functional tests shown proper functioning of the robot components. A measurement of the inspection speed in the basic, as well as in the bent, robot configuration was the first test. Speed test shows that the inspection speed is $0.2 \mathrm{~m} / \mathrm{s}$ and the robot configuration does not affect the inspection speed. Fig. 13 shows the basic and bent robot configuration speed test.

Then a test was held to adjust the geometrical parameters of the robot to suite the pipe's diameter and maximum pipeline slope. First stable position of the basic and bent robot configuration in pipe are checked. The tests showed stable positioning of the robot configuration. The inspection robot can move freely in the pipeline. The robot structure allows adjustment of geometrical parameters of the robot in pipe diameter of $160 \mathrm{~mm}$ to $300 \mathrm{~mm}$. During the tests, the maximum slope of the pipeline $\left(15^{\circ}\right)$ was determined, and this was the maximum value allowed by the power of the drives and the design of the robot. In addition, this value is enough because in the real-world working environment the pipelines with higher slope are rare. Fig. 14 shows maximum pipeline slope test bench.

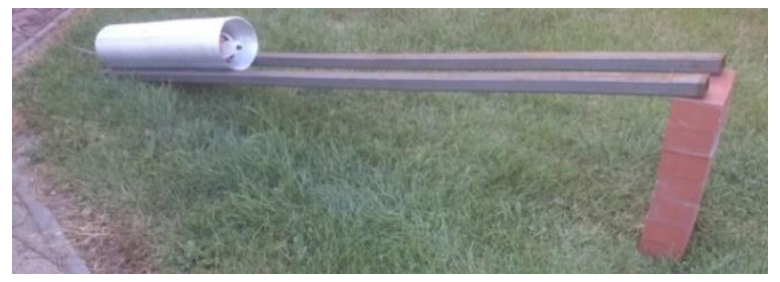

Fig. 14. The maximum pipeline slope test bench 
The applied set of image transmission allows wired and wireless image transmission. Due to the very good quality of wired transmission, it was decided to present in this work the quality of wireless transmission. Wireless image signal transmission can be used, for example, when inspecting overhead pipelines. The quality of the video data is related to the work environment, transmitter and receiver distance. The video quality test was performed in the open space. The inspection robot was placed in an aluminium pipe. Fig. 15 shows image received at $20 \mathrm{~m}$ from the video transmitter located in the robot. The test shows that a pipe was clearly visible. Based on the image it can be determined that no transmission errors occurred. The signal was lost at $50 \mathrm{~m}$ away from the robot. The designated transmission distance differs from technical data significantly. This may be the result of the failure to tune the video transmitter and video receiver.

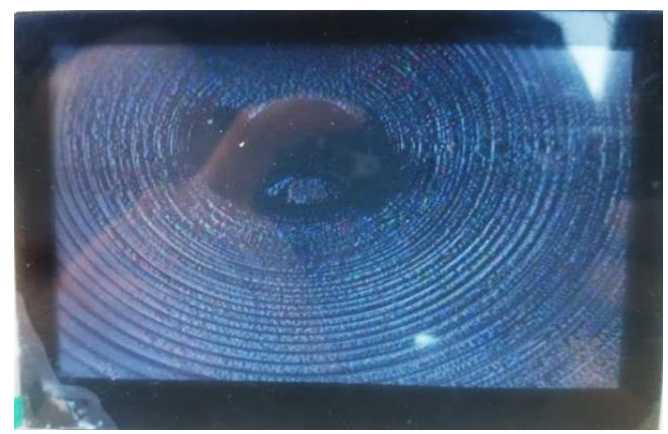

Fig. 15. View of video data transmission

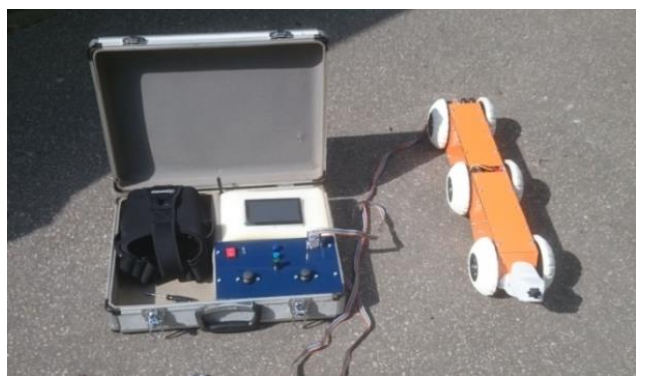

Fig. 16. The pipe inspection robot prototype and the control panel

The result of this work is a prototype of the pipe inspection mobile robot with possibility to adjust geometrical parameters to suit sewer piper diameter. The prototype of robot can inspect sewer pipelines and takes images of the pipe technical condition. The inspection system prototype is shown in Fig. 16.

\section{CONCLUSIONS}

The use of simulation tools that allow 3D simulation of components and assemblies of mechatronic devices is necessary in the complex process of product development. In this paper, the process of engineering design based on $3 \mathrm{D}$ simulation for the case of a pipe inspection robot is presented. Based on the accepted assumptions, the CAD model has been developed that would be necessary to construct the robot. The CAD model has helped to verify the operation of the proposed mechanisms and systems of the robot as well as to confirm the selection of materials and methods of making some components. The development of the device's CAD model also promotes the use of rapid prototyping to produce components, which significantly speeds up the time and reduces the cost of building a prototype. The functional tests carried out at the end allowed to determine the functional and driving parameters of the constructed model and confirmed the correct operation of robot systems, developed and made based on 3D simulations.

The use of a pneumatic actuator as an element that performs bending of the robot to adapt to the selected range of diameters is an interesting solution that meets the initial assumptions of the authors. Replacing of external electromechanical systems with pneumatic systems increases the reliability of the device and simplifies the control of the robot's matching system.

Research carried out on the prototype of the pipe inspection robot described in this article will allow the development of a commercial version of robot and further development of the presented solution.

\section{REFERENCES}

1. Baranowski L., Panasiuk J., Siwek M. (2017), Use of a Raspberry PI to built a prototype wireless control system of a mobile robot., In Proceedings of $23^{\text {rd }}$ International Conference Engineering Mechanics, 118-121.

2. Brunete A., Hernando M., Torres J.E., Gambao E. (2012), Heterogeneous multi-configurable chained microrobot for the exploration of small cavities, Automation in Construction, 21, 184-198.

3. Ciszewski M., Buratowski T., Giergiel M., Małka P., Kurc K. (2014), Virtual prototyping, design and analysis of an in-pipe inspection mobile robot, Journal of Theoretical and Applied Mechanics, 52, 417-429.

4. Erosoy S., Yilmaz Ö. (2008), The design of remote controlled bomb destruction robot implemented using mechatronics design method., Acta Mechanica et Automatica, 2(4), 11-15.

5. Fu Z., Chen Z., Zheng C., Zhao Y. (2008), A cable-tunnel inspecting robot for dangerous environment, International Journal of Advanced Robotic Systems, 5, 243-248.

6. Giergiel J., Kurc K., Szybicki D. (2015), Modular design of a robot crawler (in Polish), Modelowanie Inżynierskie, 54, 26-32.

7. Hansen P., Alismail H., Rander P., Browning B. (2015), Visual mapping for natural gas pipe inspection., International Journal of Robotics Research, 34, 532-538.

8. Kawasue K., Komatsu T. (2013), Shape measurement of a sewer pipe using a mobile robot with computer vision, International Journal of Advanced Robotic Systems, 10, 1-7.

9. Liljeback P., Pettersen K. Y., Stavdahl and Gravdahl J. T. (2012), A review on modelling, implementation, and control of snake robots, Robotics and Autonomous Systems, 60, 29-40.

10. Martinez-Gomez J., Fernandez-Caballero A., Garcia-Varea I., Rodriguez L., Romero-Gonzalez C. (2014), A taxonomy of vision systems for ground mobile robots, International Journal of Advanced Robotic Systems, 11, 1-11.

11. Ono M., Kato S. (2010), A study of an earthworm type inspection robot movable in long pipes, International Journal of Advanced Robotic Systems, 7, 85-90.

12. Panasiuk J., Siwek M. (2016), Design of inspection robot model (in Polish), Mechanik, 7, 780-781.

13. Szewczyk W. (2017), At AGH an innovative robot for inspection works is being created (in Polish), (access: 29.06.2018) https://www.agh.edu.pl/blog-naukowy/info/article/w-agh-powstajeinnowacyjny-robot-do-prac-inspekcyjnych.

14. Trebuna F., Virgala I., Pastor M., Liptak T., Mikova L. (2016), An inspection of pipe by snake robot, International Journal of Advanced Robotic Systems, 13, 1-12.

15. Yuan J., Wu X., Kang Y., Huang C. (2009), Development of an inspection robot for long-distance pipeline on-site overhaul, Industrial Robot: An International Journal, 36, 546-550. 